\title{
Solution Structure of Isoactivity Equations for Liquid-liquid Equilibrium Calculations Using the Nonrandom Two-Liquid Model
}

Zheng Li, ${ }^{\dagger}$ Kathryn A. Mumford, ${ }^{\dagger}$ Kathryn H. Smith, ${ }^{\dagger}$ Jian Chen ${ }^{\S}$ Yong Wang, ${ }^{\dagger}$ Geoffrey W. Stevens ${ }^{*},{ }^{\dagger}$

$\dagger$ Particulate Fluids Processing Centre, Department of Chemical and Biomolecular Engineering, The University of Melbourne, Victoria, 3010, Australia

§State Key Laboratory of Chemical Engineering, Tsinghua University, Beijing, 10084, PR China

Corresponding Author

*E-mail: gstevens@unimelb.edu.au. Fax: +61 383448824. 


\section{Supporting Information}

\section{Part I: Solutions of quaternary and quinary systems}

\section{Quaternary system}

The system is " $n$-Hexane (1) $+n$-Octane (2) + Benzene (3) + Sulfolane (4)" at $298.15 \mathrm{~K}$, from Chen et al. ${ }^{1}$ The initial components are: $n_{1}=0.313(0.282+0.031), n_{2}=0.183(0.171+0.012), n_{3}=0.950$ $(0.524+0.426)$ and $n_{4}=0.554(0.023+0.531)$.

Table S1. A subset of solutions for isoactivity equations in moles (quaternary system)

\begin{tabular}{|c|c|c|c|c|c|c|c|c|c|}
\hline Solutions & $n_{1}{ }^{1}$ & $n_{2}{ }^{1}$ & $n_{3}{ }^{\mathrm{I}}$ & $n_{4}{ }^{\mathrm{I}}$ & $n_{1}{ }^{\mathrm{II}}$ & $n_{2}{ }^{\mathrm{II}}$ & $n_{3}{ }^{\mathrm{II}}$ & $n_{4}{ }^{\mathrm{II}}$ & $G / R T$ \\
\hline $\mathrm{E}$ & 0.282 & 0.171 & 0.524 & 0.023 & 0.031 & 0.012 & 0.426 & 0.531 & - \\
\hline S.A & 0.278 & 0.169 & 0.525 & 0.040 & 0.035 & 0.014 & 0.425 & 0.514 & -1.510 \\
\hline S.S & 0.134 & 0.078 & 0.406 & 0.237 & 0.179 & 0.105 & 0.544 & 0.317 & -1.431 \\
\hline S.S & 0.202 & 0.118 & 0.615 & 0.358 & 0.111 & 0.065 & 0.336 & 0.196 & -1.431 \\
\hline S.S & 0.246 & 0.144 & 0.747 & 0.436 & 0.067 & 0.039 & 0.203 & 0.118 & -1.431 \\
\hline S.S & 0.147 & 0.086 & 0.447 & 0.261 & 0.166 & 0.097 & 0.503 & 0.293 & -1.431 \\
\hline
\end{tabular}

Note E represents experimental two-phase compositions.

Table S2. Solutions for isoactivity equations in mole fractions (quaternary system)

\begin{tabular}{|c|c|c|c|c|c|c|c|c|}
\hline Solutions & $x_{1}{ }^{\mathrm{I}}$ & $x_{2}{ }^{\mathrm{I}}$ & $x_{3}{ }^{\mathrm{I}}$ & $x_{4}{ }^{\mathrm{I}}$ & $x_{1}{ }^{\mathrm{II}}$ & $x_{2}{ }^{\mathrm{II}}$ & $x_{3}{ }^{\mathrm{II}}$ & $x_{4}{ }^{\mathrm{II}}$ \\
\hline $\mathrm{E}$ & 0.282 & 0.171 & 0.524 & 0.023 & 0.031 & 0.012 & 0.426 & 0.531 \\
\hline S.A & 0.274 & 0.167 & 0.519 & 0.040 & 0.036 & 0.014 & 0.430 & 0.520 \\
\hline S.S & 0.157 & 0.092 & 0.475 & 0.277 & 0.157 & 0.092 & 0.475 & 0.277 \\
\hline
\end{tabular}

Note E represents experimental two-phase compositions. 


\section{Quinary system}

The system is " $n$-hexane (1) + $n$-octane (2) + benzene (3) + toluene (4) + sulfolane (5)" at $298.15 \mathrm{~K}$, from Chen et al. ${ }^{1}$. The initial components are: $n_{1}=0.186(0.154+0.032), n_{2}=0.150(0.130+0.020)$, $n_{3}=0.245(0.133+0.112)$ and $n_{4}=0.828(0.494+0.334), n_{5}=0.590(0.088+0.502)$.

Table S3. Solutions for isoactivity equations in moles (quinary system)

\begin{tabular}{|c|c|c|c|c|c|c|c|c|c|c|c|}
\hline Solutions & $n_{1}{ }^{\mathrm{I}}$ & $n_{2}{ }^{\mathrm{I}}$ & $n_{3}{ }^{\mathrm{I}}$ & $n_{4}{ }^{\mathrm{I}}$ & $n_{5}{ }^{\mathrm{I}}$ & $n_{1}{ }^{\mathrm{I}}$ & $n_{2}{ }^{\mathrm{I}}$ & $n_{3}{ }^{\mathrm{II}}$ & $n_{4}{ }^{\mathrm{II}}$ & $n_{5}{ }^{\mathrm{Il}}$ & $G / R T$ \\
\hline E & 0.154 & 0.130 & 0.133 & 0.494 & 0.088 & 0.032 & 0.020 & 0.112 & 0.334 & 0.502 & - \\
\hline S.A & 0.155 & 0.131 & 0.139 & 0.514 & 0.094 & 0.031 & 0.019 & 0.106 & 0.314 & 0.496 & -1.915 \\
\hline S.S & 0.039 & 0.031 & 0.051 & 0.173 & 0.123 & 0.147 & 0.119 & 0.194 & 0.655 & 0.467 & -1.892 \\
\hline S.S & 0.158 & 0.127 & 0.208 & 0.702 & 0.500 & 0.028 & 0.023 & 0.037 & 0.126 & 0.090 & -1.892 \\
\hline S.S & 0.101 & 0.082 & 0.134 & 0.451 & 0.3221 & 0.085 & 0.068 & 0.111 & 0.377 & 0.268 & -1.892 \\
\hline S.S & 0.130 & 0.105 & 0.171 & 0.577 & 0.4117 & 0.0567 & 0.0457 & 0.0747 & 0.2517 & 0.179 & -1.892 \\
\hline
\end{tabular}

Note E represents experimental two-phase compositions.

Table S4. Solutions for isoactivity equations in mole fractions (quinary system)

\begin{tabular}{|c|c|c|c|c|c|c|c|c|c|c|}
\hline Solutions & $x_{1}{ }^{\mathrm{I}}$ & $x_{2}{ }^{\mathrm{I}}$ & $x_{3}{ }^{\mathrm{I}}$ & $x_{4}{ }^{\mathrm{I}}$ & $x_{5}{ }^{\mathrm{I}}$ & $x_{1}{ }^{\mathrm{II}}$ & $x_{2}{ }^{\mathrm{II}}$ & $x_{3}{ }^{\mathrm{II}}$ & $x_{4}{ }^{\text {Il }}$ & $x_{5}{ }^{\mathrm{II}}$ \\
\hline E & 0.154 & 0.130 & 0.133 & 0.495 & 0.088 & 0.032 & 0.020 & 0.112 & 0.334 & 0.502 \\
\hline S.A & 0.150 & 0.127 & 0.134 & 0.497 & 0.091 & 0.032 & 0.020 & 0.110 & 0.325 & 0.514 \\
\hline S.S & 0.093 & 0.075 & 0.123 & 0.414 & 0.295 & 0.093 & 0.075 & 0.123 & 0.414 & 0.295 \\
\hline
\end{tabular}

Note E represents experimental two-phase compositions. 


\section{Part II: Ten calculation examples}

\section{Example 1}

Ternary system: Water (1) + Phenol (2) + Benzene (3) at 298.15 K from Gonzalez et al. ${ }^{2}$.

$n_{1}=1.4238(0.4361+0.9877)$;

$n_{2}=0.4319(0.4205+0.0114)$;

$n_{3}=0.1443(0.1434+0.0009)$.

Only one correct solution is found. S.A (correct solution) occurred 14 times and S.S (symmetric solution) occurred 126 times in 1000 solutions. E represents the experimental condition.

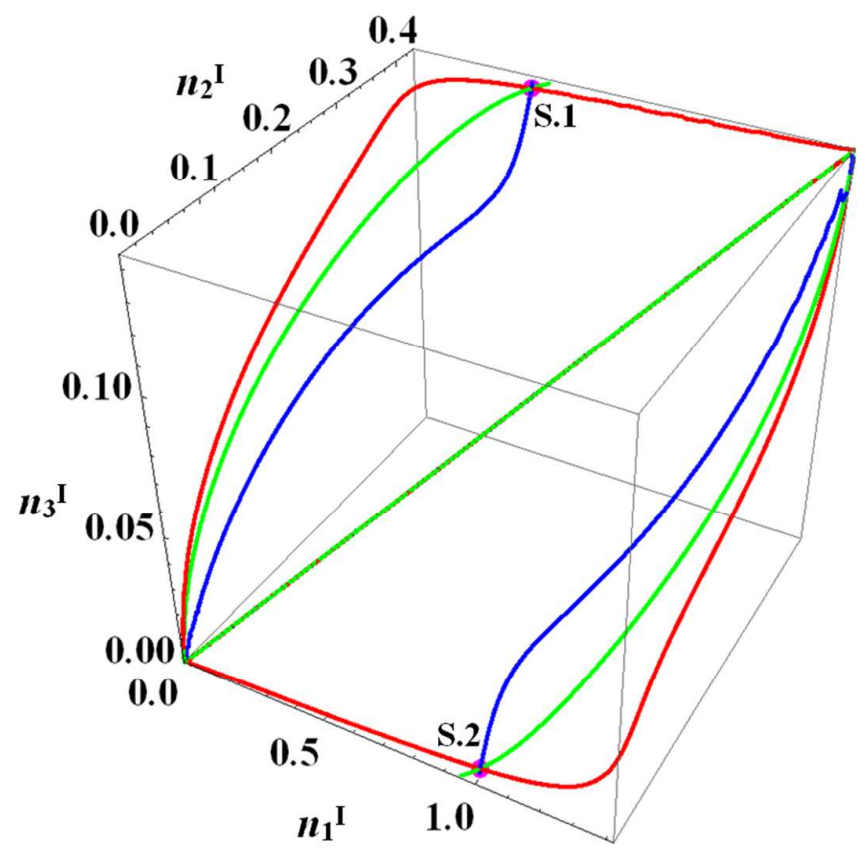

Figure S1. Solutions for isoactivity equations with the total amount of each component as constraint. S.1 and S.2 are two reversed solutions. The straight line in the middle represents symmetric solutions.

Table S1-1. Solutions for isoactivity equations in moles

\begin{tabular}{|c|c|c|c|c|c|c|c|}
\hline Solutions & $n_{1}{ }^{\mathrm{I}}$ & $n_{2}{ }^{\mathrm{I}}$ & $n_{3}{ }^{\mathrm{I}}$ & $n_{1}{ }^{\mathrm{I}}$ & $n_{2}{ }^{\mathrm{II}}$ & $n_{3}{ }^{\mathrm{II}}$ & $G / R T$ \\
\hline E & 0.4361 & 0.4205 & 0.1434 & 0.9877 & 0.0114 & 0.0009 & - \\
\hline S.A & 0.4340 & 0.4189 & 0.1426 & 0.9898 & 0.0130 & 0.0017 & -0.5671 \\
\hline S.S & 1.4006 & 0.4249 & 0.1419 & 0.0232 & 0.0070 & 0.0024 & -0.4831 \\
\hline S.S & 0.9866 & 0.2993 & 0.1000 & 0.4372 & 0.1326 & 0.0443 & -0.4831 \\
\hline S.S & 1.1378 & 0.3451 & 0.1153 & 0.2860 & 0.0868 & 0.0290 & -0.4831 \\
\hline S.S & 1.4238 & 0.4319 & 0.1443 & $\begin{array}{c}1.39 \mathrm{E}- \\
05\end{array}$ & $4.2 \mathrm{E}-06$ & $1.4 \mathrm{E}-06$ & -0.4831 \\
\hline$\ldots$ & $\ldots$ & $\ldots$ & $\ldots$ & $\ldots$ & $\ldots$ & $\ldots$ & $\ldots$ \\
\hline
\end{tabular}

Table S1-2. Solutions for isoactivity equations in mole fractions

\begin{tabular}{|c|c|c|c|c|c|c|}
\hline Solutions & $x_{1}{ }^{1}$ & $x_{2}{ }^{\mathrm{I}}$ & $x_{3}{ }^{\mathrm{I}}$ & $x_{1}{ }^{\mathrm{I}}$ & $x_{2}{ }^{\mathrm{II}}$ & $x_{3}{ }^{\mathrm{II}}$ \\
\hline E & 0.4361 & 0.4205 & 0.1434 & 0.9877 & 0.0114 & 0.0009 \\
\hline S.A & 0.4359 & 0.4208 & 0.1433 & 0.9854 & 0.0130 & 0.0017 \\
\hline S.S & 0.7119 & 0.2159 & 0.0722 & 0.7119 & 0.2159 & 0.0722 \\
\hline
\end{tabular}




\section{Example 2}

Ternary system: Acetic Acid Butyl Ester (1) + 2-Propanone (2) + 1,2-Ethanediol (3) at 304.15 K from Sørensen et al. ${ }^{3}$ (Page 410).

$n_{1}=0.44754(0.34433+0.10321)$;

$n_{2}=0.71492(0.45231+0.26261)$;

$n_{3}=0.83754(0.20336+0.63418)$.

Only one correct solution is found. S.A (correct solution) occurred 206 times and S.S (symmetric solution) occurred 143 times in 1000 solutions. E represents the experimental condition.

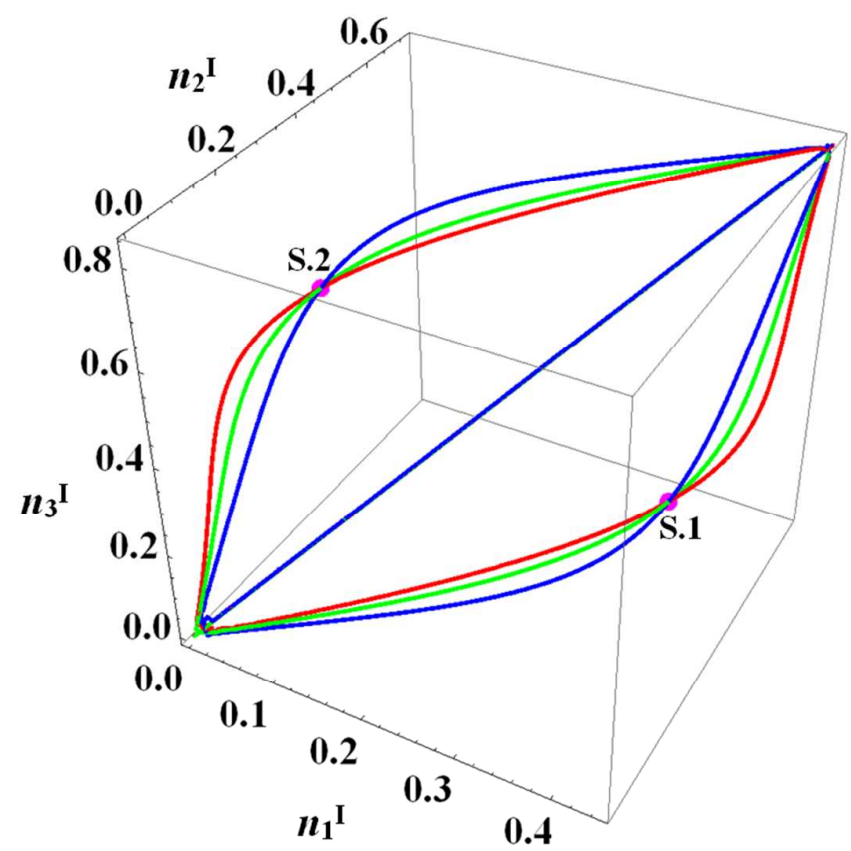

Figure S2. Solutions for isoactivity equations with the total amount of each component as constraint. S.1 and S.2 are two reversed solutions. The straight line in the middle represents symmetric solutions.

Table S2-1. Solutions for isoactivity equations in moles

\begin{tabular}{|c|c|c|c|c|c|c|c|}
\hline Solutions & $n_{1}{ }^{\mathrm{I}}$ & $n_{2}{ }^{\mathrm{I}}$ & $n_{3}{ }^{\mathrm{I}}$ & $n_{1}{ }^{\mathrm{II}}$ & $n_{2}{ }^{\mathrm{II}}$ & $n_{3}{ }^{\mathrm{II}}$ & $G / R T$ \\
\hline $\mathrm{E}$ & 0.34433 & 0.45231 & 0.20336 & 0.10321 & 0.26261 & 0.63418 & - \\
\hline S.A & 0.37262 & 0.47237 & 0.19371 & 0.07492 & 0.24255 & 0.64383 & -1.1551 \\
\hline S.S & 0.43318 & 0.69198 & 0.81067 & 0.01436 & 0.02294 & 0.02688 & -1.1389 \\
\hline S.S & 0.20014 & 0.31971 & 0.37454 & 0.24740 & 0.39521 & 0.46300 & -1.1389 \\
\hline S.S & 0.01834 & 0.02929 & 0.03432 & 0.42920 & 0.68563 & 0.80323 & -1.1389 \\
\hline S.S & 0.33995 & 0.54306 & 0.63620 & 0.10759 & 0.17186 & 0.20134 & -1.1389 \\
\hline$\ldots$ & $\ldots$ & $\ldots$ & $\ldots$ & $\ldots$ & $\ldots$ & $\ldots$ & $\ldots$ \\
\hline
\end{tabular}

Table S2-2. Solutions for isoactivity equations in mole fractions

\begin{tabular}{|c|c|c|c|c|c|c|}
\hline Solutions & $x_{1}{ }^{1}$ & $x_{2}{ }^{\top}$ & $x_{3}{ }^{1}$ & $x_{1}{ }^{\mathrm{I}}$ & $x_{2}{ }^{\mathrm{II}}$ & $x_{3}{ }^{\mathrm{II}}$ \\
\hline E & 0.34433 & 0.45231 & 0.20336 & 0.10321 & 0.26261 & 0.63418 \\
\hline S.A & 0.35874 & 0.45477 & 0.18649 & 0.07793 & 0.25232 & 0.66975 \\
\hline S.B & 0.22377 & 0.35746 & 0.41877 & 0.22377 & 0.35746 & 0.41877 \\
\hline
\end{tabular}




\section{Example 3}

Ternary system: Water (1) + Acetic Acid (2) + Ethyl Acetate (3) at $298 \mathrm{~K}$ from Colombo et al. ${ }^{4}$.

$n_{1}=1.43442(0.87819+0.55623)$;

$n_{2}=0.22615(0.07766+0.14849)$;

$n_{3}=0.33943(0.04415+0.29528)$.

Only one correct solution is found. S.A (correct solution) occurred 259 times and S.S (symmetric solution) occurred 53 times in 1000 solutions. E represents the experimental condition.

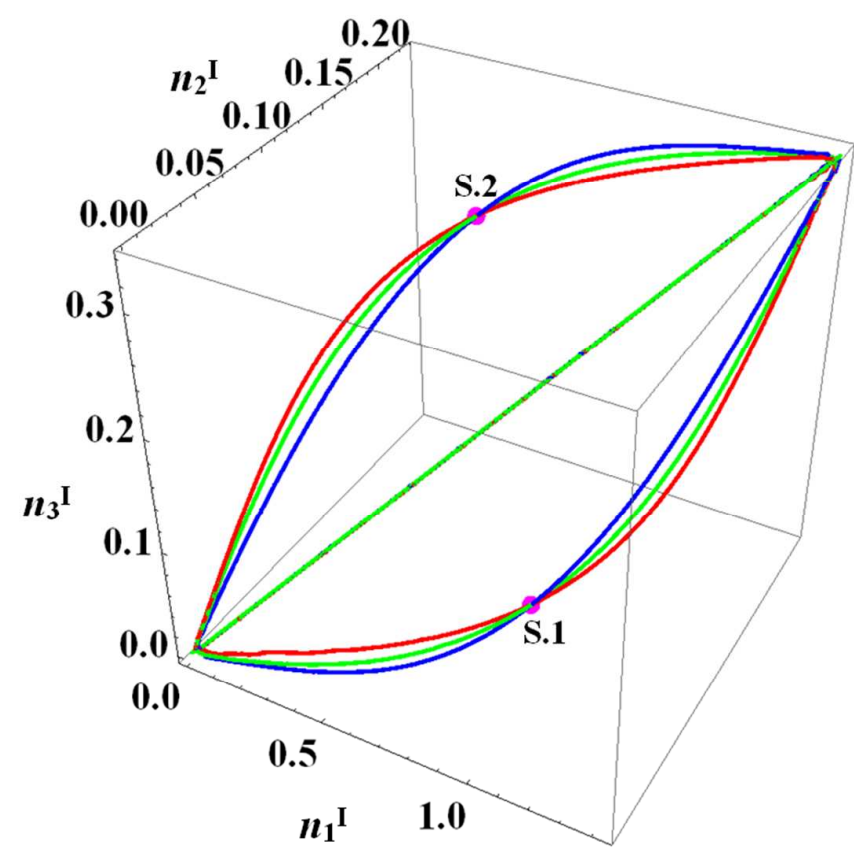

Figure S3. Solutions for isoactivity equations with the total amount of each component as constraint. S.1 and S.2 are two reversed solutions. The straight line in the middle represents symmetric solutions.

Table S3-1. Solutions for isoactivity equations in moles

\begin{tabular}{|c|c|c|c|c|c|c|c|}
\hline Solutions & $n_{1}{ }^{\mathrm{I}}$ & $n_{2}{ }^{\mathrm{I}}$ & $n_{3}{ }^{\mathrm{I}}$ & $n_{1}{ }^{\mathrm{I}}$ & $n_{2}{ }^{\mathrm{II}}$ & $n_{3}{ }^{\mathrm{II}}$ & $G / R T$ \\
\hline $\mathrm{E}$ & 0.87819 & 0.07766 & 0.04415 & 0.55623 & 0.14849 & 0.29528 & - \\
\hline S.A & 0.91852 & 0.08164 & 0.04522 & 0.51590 & 0.14451 & 0.29421 & -1.1193 \\
\hline S.S & 0.03277 & 0.00517 & 0.00776 & 1.40165 & 0.22098 & 0.33168 & -1.1073 \\
\hline S.S & 0.73670 & 0.11615 & 0.17433 & 0.69773 & 0.11000 & 0.16510 & -1.1073 \\
\hline S.S & 0.12934 & 0.02039 & 0.03061 & 1.30508 & 0.20576 & 0.30882 & -1.1073 \\
\hline S.S & 0.94156 & 0.14845 & 0.22280 & 0.49286 & 0.07770 & 0.11663 & -1.1073 \\
\hline$\ldots$ & $\ldots$ & $\ldots$ & $\ldots$ & $\ldots$ & $\ldots$ & $\ldots$ & $\ldots$ \\
\hline
\end{tabular}

Table S3-2. Solutions for isoactivity equations in mole fractions

\begin{tabular}{|c|c|c|c|c|c|c|}
\hline Solutions & $x_{1}{ }^{1}$ & $x_{2}{ }^{\top}$ & $x_{3}{ }{ }$ & $x_{1}{ }^{\mathrm{I}}$ & $x_{2}{ }^{\mathrm{II}}$ & $x_{3}{ }^{\mathrm{Il}}$ \\
\hline E & 0.87819 & 0.07766 & 0.04415 & 0.55623 & 0.14849 & 0.29528 \\
\hline S.A & 0.87865 & 0.07810 & 0.04326 & 0.54043 & 0.15138 & 0.30820 \\
\hline S.S & 0.71721 & 0.11308 & 0.16972 & 0.71721 & 0.11308 & 0.16972 \\
\hline
\end{tabular}




\section{Example 4}

Ternary system: Quinoline (1) + Furfural (2) + Water (3) at $298.15 \mathrm{~K}$ from Sørensen et al. ${ }^{5}$ (Page 208).

$n_{1}=0.15117(0.15058+0.00059) ;$

$n_{2}=0.45748(0.44457+0.01291)$;

$n_{3}=1.39135(0.40485+0.98650)$.

Only one correct solution is found. S.A (correct solution) occurred 12 times and S.S (symmetric solution) occurred 89 times in 1000 solutions. E represents the experimental condition.

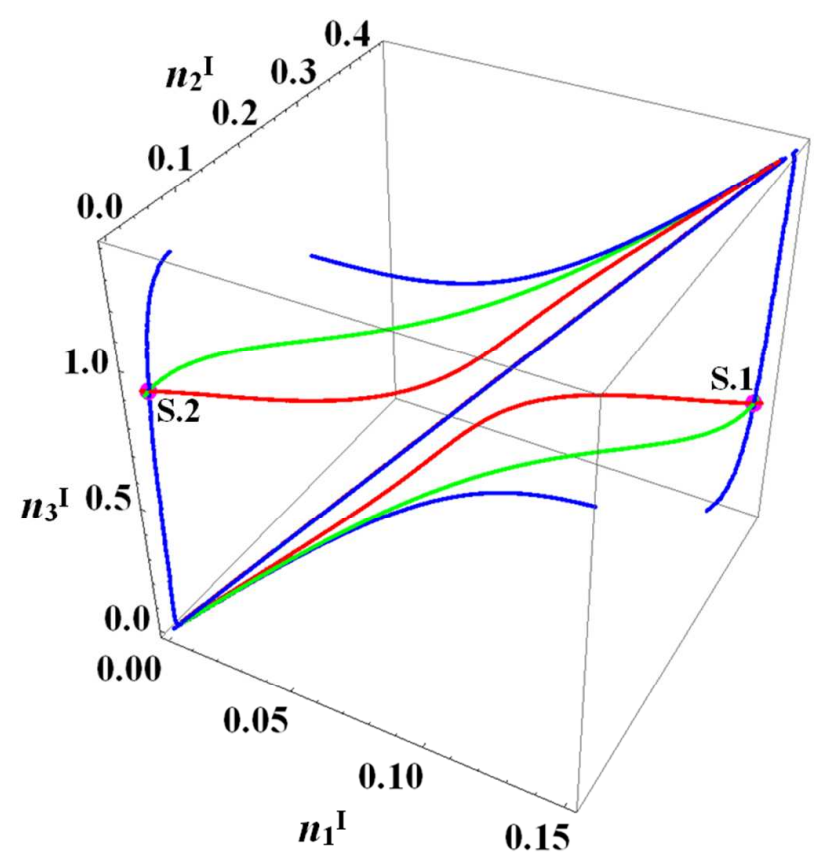

Figure S4. Solutions for isoactivity equations with the total amount of each component as constraint. S.1 and S.2 are two reversed solutions. The straight line in the middle represents symmetric solutions.

Table S4-1. Solutions for isoactivity equations in moles

\begin{tabular}{|c|c|c|c|c|c|c|c|}
\hline Solutions & $n_{1}{ }^{\mathrm{I}}$ & $n_{2}{ }^{\mathrm{I}}$ & $n_{3}{ }^{\mathrm{I}}$ & $n_{1}{ }^{\mathrm{II}}$ & $n_{2}{ }^{\mathrm{II}}$ & $n_{3}{ }^{\mathrm{II}}$ & $G / R T$ \\
\hline $\mathrm{E}$ & 0.15058 & 0.44457 & 0.40485 & 0.00059 & 0.01291 & 0.98650 & - \\
\hline S.A & 0.14832 & 0.45245 & 0.46869 & 0.00285 & 0.00503 & 0.92266 & -0.5825 \\
\hline S.S & 0.15063 & 0.45584 & 1.38637 & 0.00054 & 0.00164 & 0.00498 & -0.4744 \\
\hline S.S & 0.05835 & 0.17658 & 0.53704 & 0.09282 & 0.28090 & 0.85431 & -0.4744 \\
\hline S.S & 0.11921 & 0.36077 & 1.09723 & 0.03196 & 0.09671 & 0.29412 & -0.4744 \\
\hline S.S & 0.04155 & 0.12573 & 0.38238 & 0.10963 & 0.33175 & 1.00897 & -0.4744 \\
\hline$\ldots$ & $\ldots$ & $\ldots$ & $\ldots$ & $\ldots$ & $\ldots$ & $\ldots$ & $\ldots$ \\
\hline
\end{tabular}

Table S4-2. Solutions for isoactivity equations in mole fractions

\begin{tabular}{|c|c|c|c|c|c|c|}
\hline Solutions & $x_{1}{ }^{1}$ & $x_{2}{ }^{\top}$ & $x_{3}{ }^{\top}$ & $x_{1}{ }^{\mathrm{I}}$ & $x_{2}{ }^{\mathrm{II}}$ & $x_{3}{ }^{\mathrm{Il}}$ \\
\hline E & 0.15058 & 0.44457 & 0.40485 & 0.00059 & 0.01291 & 0.98650 \\
\hline S.A & 0.13869 & 0.42307 & 0.43825 & 0.00306 & 0.00540 & 0.99154 \\
\hline S.S & 0.07559 & 0.22874 & 0.69568 & 0.07559 & 0.22874 & 0.69568 \\
\hline
\end{tabular}




\section{Example 5}

Ternary system: Water (1) + Ethanol (2) + 1,1-Difluorothane (3) at $223.2 \mathrm{~K}$ and $6.08 \mathrm{Mpa}$ from Nakayama et al. ${ }^{6}$.

$n_{1}=0.8825(0.8157+0.0668)$;

$n_{2}=0.3096(0.1649+0.1447)$;

$n_{3}=0.8079(0.0194+0.7885)$.

Only one correct solution is found. S.A (correct solution) occurred 47 times and S.S (symmetric solution) occurred 135 times in 1000 solutions. E represents the experimental condition.

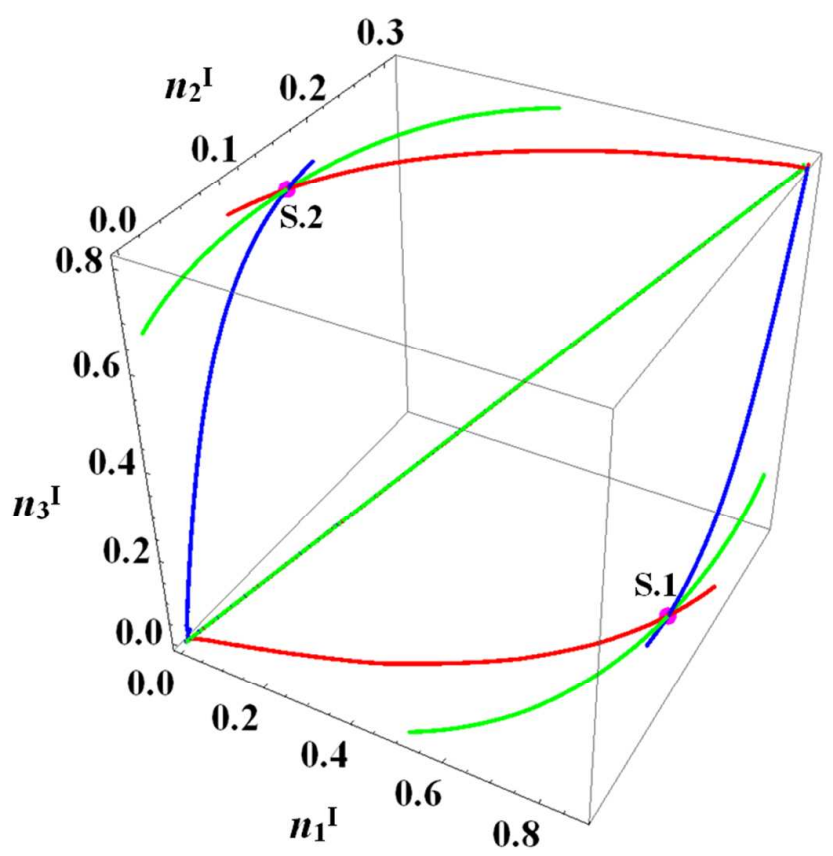

Figure S5. Solutions for isoactivity equations with the total amount of each component as constraint. S.1 and S.2 are two reversed solutions. The straight line in the middle represents symmetric solutions.

Table S5-1. Solutions for isoactivity equations in moles

\begin{tabular}{|c|c|c|c|c|c|c|c|}
\hline Solutions & $n_{1}{ }^{1}$ & $n_{2}{ }^{\mathrm{I}}$ & $n_{3}{ }^{\mathrm{I}}$ & $n_{1}{ }^{\mathrm{II}}$ & $n_{2}{ }^{\mathrm{II}}$ & $n_{3}{ }^{\mathrm{II}}$ & $G / R T$ \\
\hline E & 0.8157 & 0.1649 & 0.0194 & 0.0668 & 0.1447 & 0.7885 & - \\
\hline S.A & 0.8278 & 0.1738 & 0.0281 & 0.0547 & 0.1358 & 0.7798 & -1.1207 \\
\hline S.S & 0.0375 & 0.0132 & 0.0344 & 0.8450 & 0.2964 & 0.7735 & -0.9124 \\
\hline S.S & 0.0016 & 0.0006 & 0.0015 & 0.8809 & 0.3090 & 0.8064 & -0.9124 \\
\hline S.S & 0.6352 & 0.2229 & 0.5815 & 0.2473 & 0.0867 & 0.2264 & -0.9124 \\
\hline S.S & 0.0008 & 0.0003 & 0.0007 & 0.8817 & 0.3093 & 0.8072 & -0.9124 \\
\hline$\ldots$ & $\ldots$ & $\ldots$ & $\ldots$ & $\ldots$ & $\ldots$ & $\ldots$ & $\ldots$ \\
\hline
\end{tabular}

Table S5-2. Solutions for isoactivity equations in mole fractions

\begin{tabular}{|c|c|c|c|c|c|c|}
\hline Solutions & $x_{1}{ }^{1}$ & $x_{2}{ }^{\top}$ & $x_{3}{ }^{\top}$ & $x_{1}{ }^{\mathrm{I}}$ & $x_{2}{ }^{\mathrm{II}}$ & $x_{3}{ }^{\mathrm{Il}}$ \\
\hline E & 0.8157 & 0.1649 & 0.0194 & 0.0668 & 0.1447 & 0.7885 \\
\hline S.A & 0.8039 & 0.1688 & 0.0273 & 0.0563 & 0.1400 & 0.8037 \\
\hline S.S & 0.4413 & 0.1548 & 0.4040 & 0.4413 & 0.1548 & 0.4040 \\
\hline
\end{tabular}




\section{Example 6}

Quaternary system: 1-octanol (1) + TAME (2) + Water (3) + Methanol (4) at $298.15 \mathrm{~K}$ from Arce et $a l^{7}$.

$n_{1}=0.1664 \quad(0.1651+0.0013)$;

$n_{2}=0.1930 \quad(0.1849+0.0081)$;

$n_{3}=1.0385 \quad(0.3243+0.7142)$;

$n_{4}=0.6022 \quad(0.3258+0.2764)$.

Parameters are from Table 4 of the paper ${ }^{7}$. Only one correct solution is found. S.A (correct solution) occurred 14 times and S.S (symmetric solution) occurred 121 times in 1000 solutions. E represents the experimental condition.

Table S6-1. Solutions for isoactivity equations in moles

\begin{tabular}{|c|c|c|c|c|c|c|c|c|c|}
\hline Solutions & $n_{1}{ }^{\mathrm{I}}$ & $n_{2}{ }^{\mathrm{I}}$ & $n_{3}{ }^{\mathrm{l}}$ & $n_{4}{ }^{\mathrm{I}}$ & $n_{1}{ }^{\mathrm{II}}$ & $n_{2}{ }^{\mathrm{II}}$ & $n_{3}{ }^{\mathrm{II}}$ & $n_{4}{ }^{\mathrm{II}}$ & $G / R T$ \\
\hline $\mathrm{E}$ & 0.1651 & 0.1849 & 0.3243 & 0.3258 & 0.0013 & 0.0081 & 0.7142 & 0.2764 & - \\
\hline S.A & 0.1657 & 0.1916 & 0.2498 & 0.4150 & 0.0007 & 0.0014 & 0.7887 & 0.1872 & -2.9304 \\
\hline S.S & 0.1624 & 0.1883 & 1.0133 & 0.5876 & 0.0040 & 0.0047 & 0.0252 & 0.0146 & -2.8955 \\
\hline S.S & 0.0136 & 0.0158 & 0.0848 & 0.0492 & 0.1528 & 0.1772 & 0.9537 & 0.5530 & -2.8955 \\
\hline S.S & 0.0098 & 0.0113 & 0.0609 & 0.0353 & 0.1566 & 0.1817 & 0.9776 & 0.5669 & -2.8955 \\
\hline S.S & 0.1577 & 0.1830 & 0.9845 & 0.5709 & 0.0087 & 0.0100 & 0.0540 & 0.0313 & -2.8955 \\
\hline$\ldots$ & $\ldots$ & $\ldots$ & $\ldots$ & $\ldots$ & $\ldots$ & $\ldots$ & $\ldots$ & $\ldots$ & $\ldots$ \\
\hline
\end{tabular}

Table S6-2. Solutions for isoactivity equations in mole fractions

\begin{tabular}{|c|c|c|c|c|c|c|c|c|}
\hline Solutions & $x_{1}{ }^{1}$ & $x_{2}{ }^{1}$ & $x_{3}{ }^{1}$ & $x_{4}{ }^{1}$ & $x_{1}{ }^{I I}$ & $x_{2}{ }^{\text {II }}$ & $x_{3}{ }^{I I}$ & $x_{4}{ }^{\text {II }}$ \\
\hline E & 0.1651 & 0.1849 & 0.3243 & 0.3258 & 0.0013 & 0.0081 & 0.7142 & 0.2764 \\
\hline S.A & 0.1622 & 0.1875 & 0.2444 & 0.4060 & 0.0007 & 0.0014 & 0.8065 & 0.1914 \\
\hline S.S & 0.0832 & 0.0965 & 0.5192 & 0.3011 & 0.0832 & 0.0965 & 0.5192 & 0.3011 \\
\hline
\end{tabular}




\section{Example 7}

Quaternary system: n-Hexane (1) + n-Octane (2) + Benzene (3) + Sulfolane (4) at 298.15 K from Chen et al. ${ }^{1}$.

$$
\begin{array}{ll}
n_{1}=0.313 & (0.282+0.031) ; \\
n_{2}=0.183 & (0.171+0.012) ; \\
n_{3}=0.950 & (0.524+0.426) ; \\
n_{4}=0.554 & (0.023+0.531),
\end{array}
$$

Only one correct solution is found. S.A (correct solution) occurred 103 times and S.S (symmetric solution) occurred 531 times in 1000 solutions. E represents the experimental condition.

Table S7-1. Solutions for isoactivity equations in moles

\begin{tabular}{|c|c|c|c|c|c|c|c|c|c|}
\hline Solutions & $n_{1}{ }^{\mathrm{I}}$ & $n_{2}{ }^{\mathrm{I}}$ & $n_{3}{ }^{\mathrm{I}}$ & $n_{4}{ }^{\mathrm{I}}$ & $n_{1}{ }^{\mathrm{II}}$ & $n_{2}{ }^{\mathrm{II}}$ & $n_{3}{ }^{\mathrm{II}}$ & $n_{4}{ }^{\mathrm{II}}$ & $G / R T$ \\
\hline $\mathrm{E}$ & 0.282 & 0.171 & 0.524 & 0.023 & 0.031 & 0.012 & 0.426 & 0.531 & - \\
\hline S.A & 0.278 & 0.169 & 0.525 & 0.040 & 0.035 & 0.014 & 0.425 & 0.514 & -1.510 \\
\hline S.S & 0.134 & 0.078 & 0.406 & 0.237 & 0.179 & 0.105 & 0.544 & 0.317 & -1.431 \\
\hline S.S & 0.202 & 0.118 & 0.615 & 0.358 & 0.111 & 0.065 & 0.336 & 0.196 & -1.431 \\
\hline S.S & 0.246 & 0.144 & 0.747 & 0.436 & 0.067 & 0.039 & 0.203 & 0.118 & -1.431 \\
\hline S.S & 0.147 & 0.086 & 0.447 & 0.261 & 0.166 & 0.097 & 0.503 & 0.293 & -1.431 \\
\hline$\ldots$ & $\ldots$ & $\ldots$ & $\ldots$ & $\ldots$ & $\ldots$ & $\ldots$ & $\ldots$ & $\ldots$ & $\ldots$ \\
\hline
\end{tabular}

Table S7-2. Solutions for isoactivity equations in mole fractions

\begin{tabular}{|c|c|c|c|c|c|c|c|c|}
\hline Solutions & $x_{1}{ }^{1}$ & $x_{2}{ }^{\top}$ & $x_{3}{ }^{\top}$ & $x_{4}{ }^{1}$ & $x_{1}{ }^{\text {II }}$ & $x_{2}{ }^{\text {II }}$ & $x_{3}{ }^{\text {II }}$ & $x_{4}{ }^{\text {II }}$ \\
\hline E & 0.282 & 0.171 & 0.524 & 0.023 & 0.031 & 0.012 & 0.426 & 0.531 \\
\hline S.A & 0.274 & 0.167 & 0.519 & 0.040 & 0.036 & 0.014 & 0.430 & 0.520 \\
\hline S.S & 0.157 & 0.092 & 0.475 & 0.277 & 0.157 & 0.092 & 0.475 & 0.277 \\
\hline
\end{tabular}




\section{Example 8}

Quaternary system: Heptane (1) + Benzene (2) + Ethylbenzene (3) + Methanol (4) at 293.15 K from García-Flores et al. ${ }^{8}$.

$n_{1}=0.7033 \quad(0.5444+0.1589)$;

$n_{2}=0.1352 \quad(0.0882+0.0470)$;

$n_{3}=0.0897 \quad(0.0592+0.0305)$;

$n_{4}=1.0718 \quad(0.3082+0.7636)$.

Only one correct solution is found. S.A (correct solution) occurred 163 times and S.S (symmetric solution) occurred 100 times in 1000 solutions. E represents the experimental condition.

Table S8-1. Solutions for isoactivity equations in moles

\begin{tabular}{|c|c|c|c|c|c|c|c|c|c|}
\hline Solutions & $n_{1}{ }^{\mathrm{I}}$ & $n_{2}{ }^{\mathrm{I}}$ & $n_{3}{ }^{\mathrm{l}}$ & $n_{4}{ }^{\mathrm{l}}$ & $n_{1}{ }^{\mathrm{Il}}$ & $n_{2}{ }^{\mathrm{Il}}$ & $n_{3}{ }^{\mathrm{II}}$ & $n_{4}{ }^{\mathrm{II}}$ & $G / R T$ \\
\hline E & 0.5444 & 0.0882 & 0.0592 & 0.3082 & 0.1589 & 0.0470 & 0.0305 & 0.7636 & - \\
\hline S.A & 0.1615 & 0.0525 & 0.0262 & 0.7726 & 0.5418 & 0.0827 & 0.0635 & 0.2993 & -0.9297 \\
\hline S.S & 0.6032 & 0.1159 & 0.0769 & 0.9192 & 0.1001 & 0.0193 & 0.0128 & 0.1526 & -0.9274 \\
\hline S.S & $1.14 \mathrm{E}-05$ & $2.19 \mathrm{E}-06$ & $1.46 \mathrm{E}-06$ & $1.74 \mathrm{E}-05$ & 0.7033 & 0.1352 & 0.0897 & 1.0718 & -0.9274 \\
\hline S.S & 0.4549 & 0.0875 & 0.0580 & 0.6933 & 0.2484 & 0.0477 & 0.0317 & 0.3785 & -0.9274 \\
\hline S.S & 0.0960 & 0.0184 & 0.0122 & 0.1463 & 0.6073 & 0.1168 & 0.0775 & 0.9255 & -0.9274 \\
\hline$\ldots$ & $\ldots$ & $\ldots$ & $\ldots$ & & $\ldots$ & $\ldots$ & $\ldots$ & $\ldots$ & $\ldots$ \\
\hline
\end{tabular}

Table S8-2. Solutions for isoactivity equations in mole fractions

\begin{tabular}{|c|c|c|c|c|c|c|c|c|}
\hline Solutions & $x_{1}^{\top}$ & $x_{2}{ }^{\top}$ & $x_{3}{ }^{1}$ & $x_{4}{ }^{1}$ & $x_{1}{ }^{I I}$ & $x_{2}$ & $x_{3}{ }^{I I}$ & $x_{4}$ II \\
\hline $\bar{E}$ & 0.5444 & 0.0882 & 0.0592 & 0.3082 & 0.1589 & 0.0470 & 0.0305 & 0.7636 \\
\hline S.A & 0.5488 & 0.0838 & 0.0644 & 0.3031 & 0.1594 & 0.0518 & 0.0258 & 0.7629 \\
\hline S.S & 0.3517 & 0.0676 & 0.0449 & 0.5359 & 0.3517 & 0.0676 & 0.0449 & 0.5359 \\
\hline
\end{tabular}




\section{Example 9}

Quinary system: Heptane (1) + Dodecane (2) + Benzene (3) + Toluene (4) + Furfural (5)" at $318.15 \mathrm{~K}$ from Kumar et al. ${ }^{9}$.

$n_{1}=0.544 \quad(0.484+0.060) ;$
$n_{2}=0.174 \quad(0.165+0.009) ;$
$n_{3}=0.188 \quad(0.106+0.082) ;$
$n_{4}=0.171 \quad(0.106+0.065) ;$
$n_{5}=0.924 \quad(0.139+0.785)$.

Only one correct solution is found. S.A (correct solution) occurred 158 times and S.S (symmetric solution) occurred 438 times in 1000 solutions. E represents the experimental condition.

Table S9-1. Solutions for isoactivity equations in moles

\begin{tabular}{|c|c|c|c|c|c|c|c|c|c|c|c|}
\hline Solutions & $n_{1}{ }^{\mathrm{I}}$ & $n_{2}{ }^{\mathrm{I}}$ & $n_{3}{ }^{\mathrm{I}}$ & $n_{4}{ }^{\mathrm{I}}$ & $n_{5}{ }^{\mathrm{I}}$ & $n_{1}{ }^{\mathrm{II}}$ & $n_{2}{ }^{\mathrm{II}}$ & $n_{3}{ }^{\mathrm{II}}$ & $n_{4}{ }^{\mathrm{II}}$ & $n_{5}{ }^{\mathrm{II}}$ & $G / R T$ \\
\hline $\mathrm{E}$ & 0.484 & 0.165 & 0.106 & 0.106 & 0.139 & 0.060 & 0.009 & 0.082 & 0.065 & 0.785 & - \\
\hline S.A & 0.475 & 0.162 & 0.0971 & 0.095 & 0.122 & 0.069 & 0.012 & 0.091 & 0.076 & 0.8022 & -1.539 \\
\hline S.S & 0.041 & 0.013 & 0.014 & 0.013 & 0.070 & 0.503 & 0.161 & 0.174 & 0.158 & 0.854 & -1.475 \\
\hline S.S & 0.030 & 0.010 & 0.010 & 0.009 & 0.051 & 0.514 & 0.1644 & 0.178 & 0.162 & 0.873 & -1.475 \\
\hline S.S & 0.036 & 0.012 & 0.013 & 0.011 & 0.062 & 0.508 & 0.162 & 0.175 & 0.160 & 0.862 & -1.475 \\
\hline S.S & 0.351 & 0.112 & 0.121 & 0.110 & 0.596 & 0.193 & 0.0622 & 0.067 & 0.061 & 0.328 & -1.475 \\
\hline$\ldots$ & $\ldots$ & $\ldots$ & $\ldots$ & $\ldots$ & $\ldots$ & $\ldots$ & $\ldots$ & $\ldots$ & $\ldots$ & $\ldots$ & $\ldots$ \\
\hline
\end{tabular}

Table S9-2. Solutions for isoactivity equations in mole fractions

\begin{tabular}{|c|c|c|c|c|c|c|c|c|c|c|}
\hline Solutions & $x_{1}{ }^{1}$ & $x_{2}{ }^{1}$ & $x_{3}{ }^{1}$ & $x_{4}{ }^{\mathrm{I}}$ & $x_{5}{ }^{\mathrm{I}}$ & $x_{1}{ }^{\mathrm{II}}$ & $x_{2}{ }^{\mathrm{II}}$ & $x_{3}{ }^{\mathrm{II}}$ & $x_{4}{ }^{\mathrm{II}}$ & $x_{5}{ }^{\mathrm{II}}$ \\
\hline E & 0.484 & 0.165 & 0.106 & 0.106 & 0.139 & 0.060 & 0.009 & 0.082 & 0.065 & 0.784 \\
\hline S.A & 0.499 & 0.170 & 0.102 & 0.100 & 0.128 & 0.065 & 0.012 & 0.087 & 0.072 & 0.764 \\
\hline S.S & 0.272 & 0.087 & 0.094 & 0.085 & 0.462 & 0.272 & 0.087 & 0.094 & 0.085 & 0.462 \\
\hline
\end{tabular}




\section{Example 10}

Quinary system: Hexane (1) + Heptane (2) + Toluene (3) + m-xylene (4) + Sulfolane (5) at 298.15 K from Chen et al. ${ }^{10}$.

$n_{1}=0.295(0.280+0.015)$;

$n_{2}=0.158(0.150+0.008)$;

$n_{3}=0.247 \quad(0.171+0.076)$;

$n_{4}=0.395(0.364+0.131)$;

$n_{5}=0.805(0.035+0.770)$.

Only one correct solution is found. S.A (correct solution) occurred 37 times and S.S (symmetric solution) occurred 505 times in 1000 solutions. E represents the experimental condition.

Table S10-1. Solutions for isoactivity equations in moles

\begin{tabular}{|c|c|c|c|c|c|c|c|c|c|c|c|}
\hline Solutions & $n_{1}{ }^{\mathrm{I}}$ & $n_{2}{ }^{\mathrm{I}}$ & $n_{3}{ }^{\mathrm{I}}$ & $n_{4}{ }^{\mathrm{I}}$ & $n_{5}{ }^{\mathrm{I}}$ & $n_{1}{ }^{\mathrm{II}}$ & $n_{2}{ }^{\mathrm{II}}$ & $n_{3}{ }^{\mathrm{II}}$ & $n_{4}{ }^{\mathrm{II}}$ & $n_{5}{ }^{\mathrm{II}}$ & $G / R T$ \\
\hline $\mathrm{E}$ & 0.280 & 0.150 & 0.171 & 0.364 & 0.035 & 0.015 & 0.008 & 0.086 & 0.131 & 0.770 & \\
\hline S.A & 0.285 & 0.153 & 0.238 & 0.478 & 0.777 & 0.010 & 0.005 & 0.009 & 0.017 & 0.028 & -1.687 \\
\hline S.S & 0.224 & 0.120 & 0.187 & 0.376 & 0.611 & 0.071 & 0.038 & 0.060 & 0.119 & 0.194 & -1.529 \\
\hline S.S & 0.185 & 0.099 & 0.155 & 0.311 & 0.505 & 0.110 & 0.059 & 0.092 & 0.184 & 0.300 & -1.529 \\
\hline S.S & 0.062 & 0.033 & 0.052 & 0.104 & 0.169 & 0.233 & 0.125 & 0.195 & 0.391 & 0.636 & -1.529 \\
\hline S.S & 0.123 & 0.066 & 0.103 & 0.207 & 0.336 & 0.172 & 0.092 & 0.1441 & 0.288 & 0.469 & -1.529 \\
\hline$\ldots$ & $\ldots$ & $\ldots$ & $\ldots$ & $\ldots$ & $\ldots$ & $\ldots$ & $\ldots$ & $\ldots$ & $\ldots$ & $\ldots$ & $\ldots$ \\
\hline
\end{tabular}

Table S10-2. Solutions for isoactivity equations in mole fractions

\begin{tabular}{|c|c|c|c|c|c|c|c|c|c|c|}
\hline Solutions & $x_{1}{ }^{\mathrm{I}}$ & $x_{2}{ }^{\mathrm{I}}$ & $x_{3}{ }^{\mathrm{I}}$ & $x_{4}{ }^{\mathrm{I}}$ & $x_{5}{ }^{\mathrm{I}}$ & $x_{1}{ }^{\mathrm{II}}$ & $x_{2}{ }^{\mathrm{II}}$ & $x_{3}{ }^{\mathrm{II}}$ & $x_{4}{ }^{\mathrm{II}}$ & $x_{5}{ }^{\mathrm{II}}$ \\
\hline $\mathrm{E}$ & 0.280 & 0.150 & 0.171 & 0.364 & 0.035 & 0.015 & 0.008 & 0.086 & 0.131 & 0.770 \\
\hline S.A & 0.287 & 0.156 & 0.170 & 0.350 & 0.037 & 0.017 & 0.007 & 0.080 & 0.152 & 0.744 \\
\hline S.S & 0.148 & 0.079 & 0.124 & 0.248 & 0.403 & 0.148 & 0.079 & 0.124 & 0.248 & 0.403 \\
\hline
\end{tabular}




\section{References}

(1). Chen, J.; Duan, L.-P.; Mi, J.-G.; Fei, W.-Y.; Li, Z.-C., Liquid-liquid equilibria of multi-component systems including n-hexane, n-octane, benzene, toluene, xylene and sulfolane at $298.15 \mathrm{~K}$ and atmospheric pressure. Fluid Phase Equilib. 2000, 173, (1), 109-119.

(2). Alvarez Gonzalez, J. R.; Macedo, E. A.; Soares, M. E.; Medina, A. G., Liquid-liquid equilibria for ternary systems of water-phenol and solvents: data and representation with models. Fluid Phase Equilib. 1986, 26, (3), 289-302.

(3). Sørensen, J. M.; Arlt, W., Liquid-liquid equilibrium data collection-ternary systems. DECHEMA: Frankfurt, 1980.

(4). Colombo, A.; Battilana, P.; Ragaini, V.; Bianchi, C. L.; Carvoli, G., Liquid-Liquid Equilibria of the Ternary Systems Water + Acetic Acid + Ethyl Acetate and Water + Acetic Acid + Isophorone (3,5,5-Trimethyl2-cyclohexen-1-one). J. Chem. Eng. Data 1999, 44, (1), 35-39.

(5). Sørensen, J. M.; Arlt, W., Liquid-liquid equilibrium data collection-ternary and quaternary systems. DECHEMA: Frankfurt, 1980.

(6). Nakayama, T.; Sagara, H.; Arai, K.; Saito, S., High pressure liquid-liquid equilibria for the system of water, ethanol and 1,1-difluoroethane at 323.2 K. Fluid Phase Equilib. 1987, 38, (1-2), 109-127.

(7). Arce, A.; Blanco, M.; Soto, A., Determination and correlation of liquid-liquid equilibrium data for the quaternary system 1-octanol+2-methoxy-2-methylbutane+water+methanol at $25^{\circ} \mathrm{C}$. Fluid Phase Equilib. 1999, 158-160, (0), 949-960.

(8). García-Flores, B. E.; Águila-Hernández, J.; García-Sánchez, F.; Aquino-Olivos, M. A., (Liquid-liquid) equilibria for ternary and quaternary systems of representative compounds of gasoline + methanol at 293.15K: Experimental data and correlation. Fluid Phase Equilib. 2013, 348, (0), 60-69.

(9). Kumar, U. K. A.; Mohan, R., Quinary and Eight-Component Liquid-Liquid Equilibria of Mixtures of Alkanes, Aromatics, and Solvent (Furfural). J. Chem. Eng. Data 2013, 58, (8), 2194-2201.

(10). Chen, J.; Mi, J.; Fei, W.; Li, Z., Liquid-liquid equilibria of quaternary and quinary systems including sulfolane at 298.15 K. J. Chem. Eng. Data 2001, 46, (1), 169-171. 